\title{
Switching Monopolar No-Touch Radiofrequency Ablation Using Octopus Electrodes for Small Hepatocellular Carcinoma: A Randomized Clinical Trial
}

\author{
Sae-Jin Park ${ }^{a}$ Eun Ju Choc Jeong-Hoon Lee ${ }^{c}$ Su Jong $\mathrm{Yu}^{\mathrm{c}}$ Yoon Jun Kim ${ }^{\mathrm{c}}$ \\ Jung-Hwan Yoon ${ }^{c}$ Hyo-Jin Kang ${ }^{a}$ Jeong Hee Yoon ${ }^{a, b}$ Dong Ho Lee ${ }^{a, b}$ \\ Se Hyung Kimª, b, Jae Young Lee ${ }^{a, b}$ Jeong Min Lee ${ }^{a, b, d}$ \\ a Department of Radiology, Seoul National University Hospital, Seoul, Republic of Korea; b Department of Radiology,

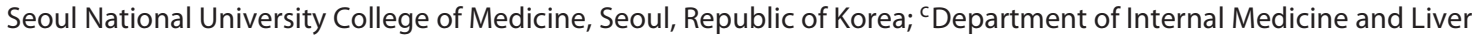 \\ Research Institute, College of Medicine, Seoul National University, Seoul, Republic of Korea; ${ }^{d}$ Institute of Radiation \\ Medicine, Seoul National University Medical Research Center, Seoul, Republic of Korea
}

\section{Key Results}

- A sufficient ablative margin was more frequently achieved in the no-touch RFA group $(57 / 60=95 \%)$ than in the conventional RFA group $(50 / 64=78.1 \%)$ on immediate follow-up CT $(p=0.02,95 \%$ CI $=$ $0.02-0.26)$.

- The cumulative incidence of local tumor progression in the no-touch RFA group was significantly lower than that in the conventional RFA group $(p=0.02, \mathrm{HR}=3.95,95 \% \mathrm{CI}=1.2-12.9)$.

- In the Cox regression model, conventional RFA was the predictive factor for local tumor progression $(p=0.04, \mathrm{HR}=4.95,95 \% \mathrm{CI}=1.1-23)$.

\section{Keywords}

Hepatocellular carcinoma · Radiofrequency ablation · Local tumor progression

\section{Abstract \\ Introduction: A switching monopolar no-touch radiofre- quency ablation (RFA) technique is used for small hepatocel- lular carcinoma (HCC); however, there have not been any randomized clinical trials comparing this technique to the conventional RFA technique. Objective: This study aims to compare the results of two RFA techniques, and to compara- tively identify more effective methods to reduce the pro-}

gression of local tumors associated with small HCC $(\leq 2.5$ $\mathrm{cm})$. Methods: This prospective randomized clinical trial (NCT03375281) recruited a total of 116 participants (M:F, 93: $23 ; 68.3 \pm 8.4$ years) between October 2016 and September 2017. The primary outcome was the cumulative incidence of local tumor progression (LTP) after RFA. Secondary outcomes included technical success rate, technique efficacy, and RFA procedure characteristics. Kaplan-Meier analysis and the Cox proportional hazard regression model were used. Results: The mean follow-up period was 24.1 months. A sufficient ablative margin was more frequently achieved in the no-touch RFA group $(57 / 60=95 \%)$ than in the conventional RFA group $(50 / 64=78.1 \%)$ on immediate follow-up CT
(C) 2020 The Author(s)

Published by S. Karger AG, Basel

This is an Open Access article licensed under the Creative Commons Attribution-NonCommercial-4.0 International License (CC BY-NC) (http://www.karger.com/Services/OpenAccessLicense), applicable to the online version of the article only. Usage and distribution for commercial purposes requires written permission.
Jeong Min Lee, MD

Department of Radiology, Seoul National University Hospital

101 Daehak-ro, Jongno-gu

Seoul 03080 (Republic of Korea)

jmsh@snu.ac.kr 
( $p=0.01$ ). The cumulative incidence of LTP in the no-touch RFA group was significantly lower than that in the conventional RFA group $(p=0.02)$. In multivariable analysis, notouch RFA was the only predictive factor for LTP ( $p=0.04$, hazard ratio $=0.2,95 \%$ confidence interval $=0.04-0.94$ ). Conclusions: A switching monopolar no-touch RFA technique is a favorable treatment option and provides lower LTP after RFA compared with conventional RFA for small HCC.

(c) 2020 The Author(s)

Published by S. Karger AG, Basel

\section{Introduction}

There has been a recent increase in the use of screening tests for high-risk groups of hepatocellular carcinoma (HCC), along with an increase in the proportion of small HCC [1]. Curative treatment options for small HCC include liver transplantation, hepatic resection, microwave ablation, and radiofrequency ablation (RFA) [2]. RFA is primarily used as a noninvasive local therapeutic modality for HCC, which is difficult to treat surgically [3].

RFA is performed by penetrating a tumor by inserting radiofrequency (RF) electrodes, using one or more RF electrodes depending on the size of the tumor and the desired ablation zone. It is suggested that inserting an electrode to concentrate high-frequency energy in the center of the tumor may enhance the therapeutic effect [4]. However, an important limitation of conventional RFA is that the local tumor progression (LTP) is more than $10 \%$ $[5,6]$. Furthermore, it may spread the tumor along the insertion route of the electrode. In previous studies where RFA was performed, track seeding was observed in $0.2-$ $0.5 \%$ of patients due to the dispersal of tumor cells $[7,8]$. Also, there is a theoretical possibility that tumors may experience bubble production within the tumor due to temperature rise of the tissue near the electrode during thermal ablation, which subsequently increases the intratumoral interstitial pressure. Then, the increased interstitial pressure within the tumor during the ablation procedure may spread the tumor cells and increase the LTP [9-12].

Therefore, a method is needed to prevent such potential tumor spread without elevating the intratumoral interstitial pressure. Besides, if ablation can be performed without penetrating the tumor, it will fundamentally prevent the spread of the tumor along the electrode insertion route.

Consequently, the "no-touch" technique was introduced, avoiding the need for incisions into tumors during surgery. In several retrospective studies, "no-touch" RFA (Nt-RFA) using multibipolar RF technology showed a similar success and complication rate, and better LTP than conventional RFA using a monopolar RF technology [13-15]. Although one prospective cohort study compared conventional RFA with Nt-RFA, a different RF ablation system and different electrodes for each RFA technique were used [16]. Therefore, it is still uncertain whether the better therapeutic outcome originated from the different RF techniques (Nt-RFA vs. conventional tumor puncture technique) or the different hardware of RFA systems. Furthermore, to our knowledge, there have been no randomized clinical trials comparing Nt-RFA with conventional RFA using the same RFA system and electrodes.

Therefore, this study aimed to compare the results of conventional RFA and Nt-RFA using a switching monopolar RF technology and to evaluate which method is more effective in reducing the LTP prospectively.

\section{Materials and Methods}

This randomized clinical trial was performed from October 2016 to September 2017 in accordance with the ethical guidelines of the 1975 Declaration of Helsinki, and the protocol was approved by the Institutional Review Board of our institution (IRB No. 1604137-758). This study was registered in ClinicalTrials.gov (https:// clinicaltrials.gov, registration No.: NCT03375281). Financial support was provided by STARmed Corp. (No. 06-2016-2370).

\section{Participants}

Between October 2016 and September 2017, participants were recruited according to the following eligibility criteria: (a) age between 20 and 85 years, (b) Child-Pugh class A, (c) number of HCCs $<3$, (d) size $\leq 2.5 \mathrm{~cm}$, (e) treatment-naïve HCC or newly developed HCC in participants who had had successful local tumor control for HCCs (within Milan criteria) by surgery, transarterial chemoembolization (TACE), RFA or percutaneous ethanol injection for at least more than 2 years, and (g) imaging within 60 days before RFA treatment. Based on our previous study [17] results showing that the minimum diameter of the ablation sphere created by switching monopolar RFA using the separable clustered electrodes (Octopus ${ }^{\circledR}$ electrode; STARmed, Goyang, Korea) was approximately $3.76 \mathrm{~cm}$, we enrolled tumors smaller than $2.5 \mathrm{~cm}$.

Exclusion criteria were as follows: (1) HCC abutting main hepatic veins or the first branches of the main portal vein $(\geq 5 \mathrm{~mm})$, (2) absence of a border under fusion image, (3) platelet count $\leq 50,000 / \mathrm{mm}^{3}$ or INR prolongation $\geq 50 \%$, (4) presence of vascular invasion or extrahepatic metastasis.

The diagnosis of HCC was made based on the presence of risk factors and typical imaging appearance at CT or MRI according to the Liver Imaging Reporting and Data System [18]. We collected clinical information such as sex, age, origin of cirrhosis, and lab data. The shape of the HCC was classified as round, oval, or irregular according to the boundary of the tumor. The segmental 


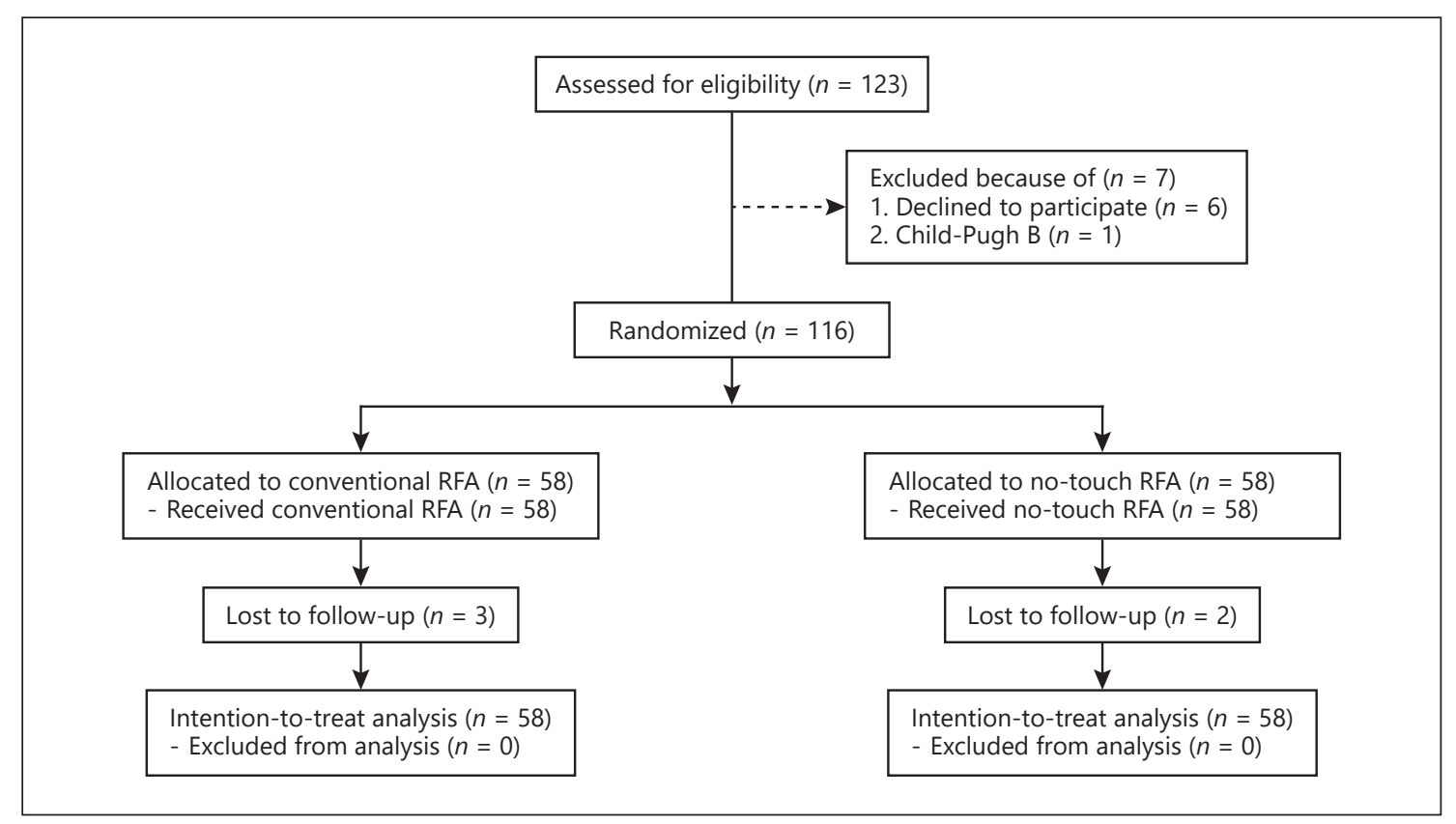

Fig. 1. Study diagram.

location of HCC was determined using the Couinaud classification [19]. Subcapsular HCC was defined as an index tumor located within $1 \mathrm{~mm}$ of the liver capsule [20]. Liver cirrhosis was diagnosed using the radiological diagnostic criteria (morphological criteria) using CT and/or MRI [2].

\section{Enrollment and Assignment}

All participants provided written informed consent before randomization. Participants who met the criteria and signed the consent forms were randomly assigned to two groups (the Nt-RFA and conventional RFA groups at a 1:1 ratio) by the stratified randomization and block randomization method beforehand, which was developed by the Medical Research Collaborating Center of our institution. This was determined by size $(<2 \mathrm{~cm}$ or $2-2.5 \mathrm{~cm})$, number (single or two), and type of HCC (naïve HCC or recurrent HCC). Group allocation was masked to the participants (Fig. 1).

\section{RFA Procedures}

The RFA system was a separable clustered electrode (Octopus ${ }^{\circledR}$ electrode; STARmed, Goyang, Korea) with 2 or 3 internally cooled electrodes, each with a 2- to 3-cm-long active tip (suppl. Appendix 1; see www.karger.com/doi/10.1159/000512338 for all online suppl. material) [21]. Percutaneous RFA procedures were conducted using real-time US-CT-MR fusion guidance (Navigator-GE \& Siemens, Samsung, PercuNav-Phillips) with conscious sedation by one of four radiologists (D.H.L, S.H.K, J.Y.L, and J.M.L) who have 12-21 years of clinical experience in RFA [22]. Electrodes were placed using a free-hand technique. The goal of RFA was to achieve complete ablation of both the visible tumor and a $0.5-$ to $1-\mathrm{cm}-$ wide ablation margin in the normal liver parenchyma surrounding the tumor [23]. We maintained each tip temperature below $25^{\circ} \mathrm{C}$ by infusing normal saline solution at $0^{\circ} \mathrm{C}$ into the lumen of each electrode using a peristaltic pump (VIVA Pump; STARmed). Grounding was achieved by attaching 4 pads to the participant's back. We continuously monitored the technical parameters, such as power output, impedance, applied currents, and total delivered energy, and recorded them using VIVA Monitor Software V 1.0 (STARmed).

In the conventional RFA procedure, 2 or 3 electrodes were placed within the target tumor with approximately $10-15 \mathrm{~mm}$ interelectrode distance, ideally within the middle zone of the target tumor. One cycle of ablation was performed for approximately $8-10 \mathrm{~min}$ in tumors $<2.5 \mathrm{~cm}$, and if an optimal ablation margin was not achieved after the first cycle of ablation, additional cycle(s) of RFA were done followed by repositioning of the electrode(s), as appropriate [23]. The precise techniques of electrode insertion using the separable clustered electrode are described elsewhere [23, 24].

Nt-RFAs were performed as described by Seror et al. [13], which involved puncturing 3 electrodes into the tumor-free area adjacent to the lesion (Fig. 2), contrary to directly puncturing the tumor as in conventional RFA (Fig. 3). When the index tumor was located on the liver surface, best efforts were made to avoid direct puncture of the tumor by taking an oblique pathway to obtain a rim of nontumorous liver tissue while placing the electrode [25].

The number of electrodes and the geometric composition of their insertion depended on the tumor size in both Nt-RFAs and conventional RFAs (suppl. Appendix 2). In most cases, all 3 electrodes were used, and when the HCC with a size of less than $2 \mathrm{~cm}$ was treated in the conventional method, 1 or 2 electrodes were placed inside the HCC, and the remaining electrodes were placed in the surrounding parenchyma. In the RFA procedure, switching monopolar mode was used in both groups [26]. The switching monopolar mode is that switching between multiple electrodes oc- 
Fig. 2. No-touch RFA of small tumor under fusion imaging guidance. A 60-year-old man with alcoholic liver cirrhosis, with a history of transarterial chemoembolization. On follow-up, there is still viable HCC in segment $5(1.6 \mathrm{~cm})$. a Arterial phase gadoxetic acid-enhanced MR image demonstrates $1.6-\mathrm{cm}$ arterial enhancing nodular lesion in segment 5 of the liver (arrow). b Under fusion imaging guidance between real-time working US and reference hepatobiliary phase MR images, location of the index tumor is determined, and the electrode is inserted. c After RF energy delivery, an echo cloud of microbubbles is created and encompasses the index tumor. d Immediate follow-up contrast-enhanced portal venous phase transverse CT image displays complete ablation of the target tumor with a sufficient ablation margin.

Fig. 3. Conventional RFA of small tumor under fusion imaging guidance. A 64-yearold man, a hepatitis B virus carrier, with a history of operation for HCC. On followup MRI, there is recurrent tumor in the segment $5(2.4 \mathrm{~cm})$ subcapsular portion. a Arterial phase gadoxetic acid-enhanced MR image demonstrates a $2.4-\mathrm{cm}$ arterial enhancing nodular lesion in segment $\mathrm{V}$ of the liver (arrow). b Under fusion imaging guidance between real-time working US and reference hepatobiliary phase MR images, location of the index tumor is determined, and the electrode is inserted. c After RF energy delivery, an echo cloud of microbubbles is created and encompasses the index tumor. d Immediate follow-up contrast-enhanced portal venous phase transverse CT image displays complete ablation of the target tumor with a sufficient ablation margin.
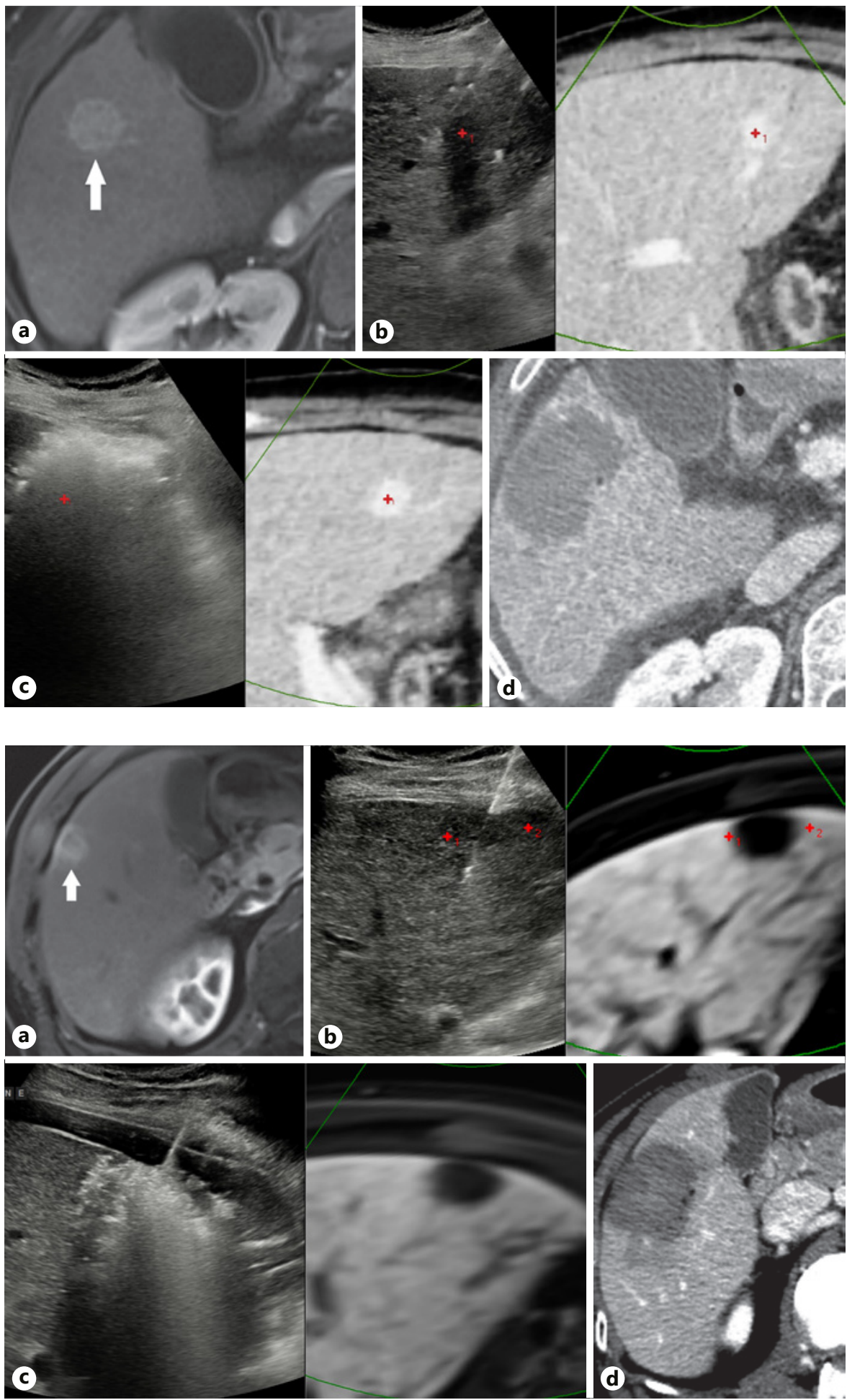

curred according to impedance changes to transfer energy through other electrically independent electrodes using the power pulse algorithm [27]. Artificial ascites (5\% dextrose water solution), used to ablate subcapsular tumors or tumors located near the diaphragm, were aspirated as much as possible after the procedures [23].

\section{Follow-Up after Treatment}

Participants with a complete response underwent imaging and measurement of serum $\alpha$-fetoprotein every 3 months during the 1 st year and every 3-6 months during the 2nd year [28]. 
Table 1. Demographics of participants ( $n$, \%)in two groups

\begin{tabular}{|c|c|c|c|c|}
\hline Category & Total & No-touch RFA & Conventional RFA & $p$ value \\
\hline \multicolumn{5}{|l|}{ Sex } \\
\hline Male & $93(80.2)$ & $45(77.6)$ & $48(82.8)$ & 0.49 \\
\hline Female & $23(19.8)$ & $13(22.4)$ & $10(17.2)$ & \\
\hline Age $^{\mathrm{a}}$, years & $68.3 \pm 8.4$ & $68.2 \pm 7.9$ & $68.4 \pm 8.9$ & 0.86 \\
\hline Origin of liver cirrhosis & & & & 0.43 \\
\hline Hepatitis B virus-related & 92 & 46 & 46 & \\
\hline Hepatitis C virus-related & 10 & 6 & 4 & \\
\hline Alcoholism & 9 & 5 & 4 & \\
\hline NBNC & 10 & 3 & 7 & \\
\hline Tumor number & & & & 0.15 \\
\hline One & 108 & 56 & 52 & \\
\hline Two & 8 & 2 & 6 & \\
\hline Previous treatment & & & & 0.31 \\
\hline TACE & 80 & 41 & 39 & \\
\hline Operation & 15 & 5 & 10 & \\
\hline RFA & 34 & 13 & 21 & \\
\hline PEIT & 8 & 4 & 4 & \\
\hline Tumor shape & & & & 0.71 \\
\hline Oval & 85 & 43 & 42 & \\
\hline Round & 31 & 13 & 18 & \\
\hline Irregular & 8 & 4 & 4 & \\
\hline Type of tumor & & & & 0.65 \\
\hline Naïve HCC & 5 & 3 & 2 & \\
\hline Recurrent HCC & 111 & 55 & 56 & \\
\hline $\begin{array}{l}\text { Tumor segmental location } \\
1,2,3,4,5,6,7,8\end{array}$ & & & & 0.86 \\
\hline Tumor location & & & & 0.52 \\
\hline Subcapsular & $17(13.7)$ & $7(11.7)$ & $10(15.6)$ & \\
\hline Central & $107(86.3)$ & $53(88.3)$ & $54(84.4)$ & \\
\hline Tumor size ${ }^{\mathrm{a}}, \mathrm{cm}$ & $1.7 \pm 0.5$ & $1.6 \pm 0.5$ & $1.7 \pm 0.5$ & 0.35 \\
\hline$<2$ & 76 & 39 & 37 & \\
\hline $2-2.5$ & 40 & 19 & 21 & \\
\hline \multicolumn{5}{|l|}{$\mathrm{Lab}$} \\
\hline Total bilirubin $^{\mathrm{a}}$ & $0.7 \pm 0.4$ & $0.6 \pm 0.4$ & $0.8 \pm 0.4$ & 0.78 \\
\hline Prothrombin time $\mathrm{a}^{\mathrm{a}}$ & $1.1 \pm 0.1$ & $1 \pm 0.1$ & $1.1 \pm 0.1$ & 0.64 \\
\hline Albumin $^{\mathrm{a}}$ & $4 \pm 0.4$ & $4 \pm 0.4$ & $4 \pm 0.4$ & 0.38 \\
\hline Platelets, $n \times 10^{3}$ & 135 & 135 & 136 & 0.88 \\
\hline Range & $48-280$ & $69-280$ & $48-272$ & \\
\hline $\mathrm{AFP}, \mathrm{ng} / \mathrm{mL}$ & 91.9 & 15.4 & 168.4 & 0.2 \\
\hline Range & $<1-6,840$ & $<1-223.5$ & $<1-6,840$ & \\
\hline Follow-up period ${ }^{\mathrm{a}}$, months & $24.1 \pm 5.5$ & $24.6 \pm 5.3$ & $23.7 \pm 5.7$ & 0.34 \\
\hline
\end{tabular}

RFA, radiofrequency ablation; NBNC, hepatitis B surface antigen and hepatitis C antibody negative; TACE, transarterial chemoembolization; HCC, hepatocellular carcinoma; PEIT, percutaneous ethanol injection therapy; AFP, a-fetoprotein. ${ }^{a}$ Mean \pm SD.

\section{Outcomes}

The primary outcome was LTP, which was defined as the reappearance of HCC adjacent to the ablated zone after successful treatment. Secondary outcomes were technical success rate, technique efficacy, and RFA procedure characteristics. RFA procedure characteristics included total procedure time, ablation time, ablation volume, sufficient ablative margin, and complications. The ablation volume was determined using the following formula: $V=$
$4 / 3 \pi \times r_{x} \times r_{y} \times r_{z}$ [29]. The term "sufficient ablative margin" was confirmed at the immediate follow-up CT and defined as $5 \mathrm{~mm}$ from the tumor margin.

Technical success was defined as full coverage of the index tumor with an ablative margin greater than or equal to $5 \mathrm{~mm}$ at immediate post-RFA CT [30]. Technique efficacy was defined as complete ablation of macroscopic tumor at 1-month follow-up CT or MRI. 
Table 2. Procedure characteristics

\begin{tabular}{|c|c|c|c|}
\hline Category & No-touch RFA & Conventional RFA & $p$ value \\
\hline Total procedure time ${ }^{\mathrm{a}}$, min & $47.4 \pm 28$ & $45.2 \pm 13$ & 0.59 \\
\hline Ablation time ${ }^{\mathrm{a}}$, min & $12.2 \pm 6.1$ & $12.1 \pm 5.6$ & 0.9 \\
\hline Ablation volume $\mathrm{a}^{\mathrm{a}} \mathrm{cm}^{3}$ & $50.3 \pm 31.6$ & $56.2 \pm 32.4$ & 0.32 \\
\hline Energy $^{\mathrm{a}}, \mathrm{kcal}$ & $14.5 \pm 9.9$ & $15.6 \pm 8$ & 0.5 \\
\hline Hospital stay ${ }^{\mathrm{a}}$, day & $1.5 \pm 1$ & $1.8 \pm 3$ & 0.99 \\
\hline Sufficient safety margin ${ }^{b}$ & & & 0.01 \\
\hline Sufficient & $57(95 \%)$ & $50(78.1 \%)$ & \\
\hline Insufficient & 3 & 14 & \\
\hline Artificial ascites infusion & $36(62.1 \%)$ & $38(65.5 \%)$ & 0.7 \\
\hline Complication & $7(12.1 \%)$ & $6(10.3 \%)$ & 0.77 \\
\hline Ascites & 6 & 6 & \\
\hline Pseudoaneurysm & 1 & 0 & \\
\hline Bowel perforation & 0 & 1 & \\
\hline Technical success & $60(100 \%)$ & $64(100 \%)$ & \\
\hline Technique efficacy & $59(98.3 \%)$ & $64(100 \%)$ & 0.3 \\
\hline RF mode & & & 0.56 \\
\hline Switching monopolar & 58 & 58 & \\
\hline
\end{tabular}

RFA, radiofrequency ablation. Technical success was achieved in all patients. Two major complications of postprocedural pseudoaneurysm and bowel perforation occurred. ${ }^{a}$ Mean $\pm \mathrm{SD}$. ${ }^{\mathrm{b}}$ Confirmed at immediate follow-up CT.

\section{Statistical Analysis}

The sample size was calculated using a reported cumulative incidence of LTP of $6-7 \%$ after Nt-RFA and $20-30 \%$ after conventional RFA $[14,31]$. A total of 56 participants were required in each group to achieve a power of $80 \%$ and an $\alpha$-level of 0.05 for a 2 -sided statistically significant difference. We included 61-62 participants in each RFA group to allow an expected $10 \%$ dropout rate after randomization.

Intergroup demographic data, technical success, technique efficacy, and RFA procedure characteristics were analyzed by the Mann-Whitney test and by the Fisher exact test. Survival curves and cumulative incidence of LTP were generated by the KaplanMeier method and compared by the log-rank test. To determine the significant risk factors predicting LTP, we used Cox proportional hazards regression models and the Wald test.

All statistical tests were 2-sided, and the null hypothesis was rejected when $p<0.05$. The statistical analyses were performed using MedCalc statistical software, version 19.1 (MedCalc Software, Mariakerke, Belgium).

\section{Results}

\section{Demographic of Study Participants}

Between October 2016 and September 2017, 123 participants were assessed for eligibility, of which 6 refused to participate and 1 did not meet the inclusion criteria before randomization. A total of 116 participants met the criteria and were randomly assigned to each group in a
1:1 ratio. In both the conventional and Nt-RFA groups, all participants received treatments as assigned. The study was completed on August 8, 2019. The mean follow-up period was 24.1 months. Three of 58 participants (5.2\%) in the conventional RFA group and 2 of 58 participants (3.4\%) in the Nt-RFA group were lost to follow-up (Fig. 1). The demographic data of the participants are presented in Table 1. There were 93 male participants. Among the etiology of liver cirrhosis, hepatitis B virusrelated cases comprised the largest portion. There were 108 participants with one HCC and 8 participants with two HCCs. Eighty participants underwent TACE, and 34 participants underwent RFA as previous treatments. There were no target lesions due to LTP after TACE or RFA. Among the 80 participants who underwent TACE, 41 participants were enrolled in the Nt-RFA group. Five participants had naïve HCC. There were 76 HCCs under $2 \mathrm{~cm}$, and 17 participants had subcapsular tumor locations. There was no significant difference between the two groups in the demographic data.

\section{Procedure Characteristics}

The procedure characteristics in the two groups are presented in Table 2. Technical success was achieved in all tumors. Technique efficacy was achieved in 59 (98.3\%, Nt-RFA) and 64 (100\%, conventional RFA) participants. 
Fig. 4. Local tumor progression after conventional RFA. A 53-year-old man, a hepatitis $\mathrm{B}$ virus carrier, underwent conventional RFA for HCC in segment 7. On follow-up CT, there is HCC in segment 7 (arrows), at the margin of the treated lesion. After CT scan, he underwent additional TACE. a Arterial phase (before RFA). b Delay phase (before RFA). c Immediately after RFA. d Arterial phase (after RFA). e Portal phase (after RFA). f Coronal reconstruction image.

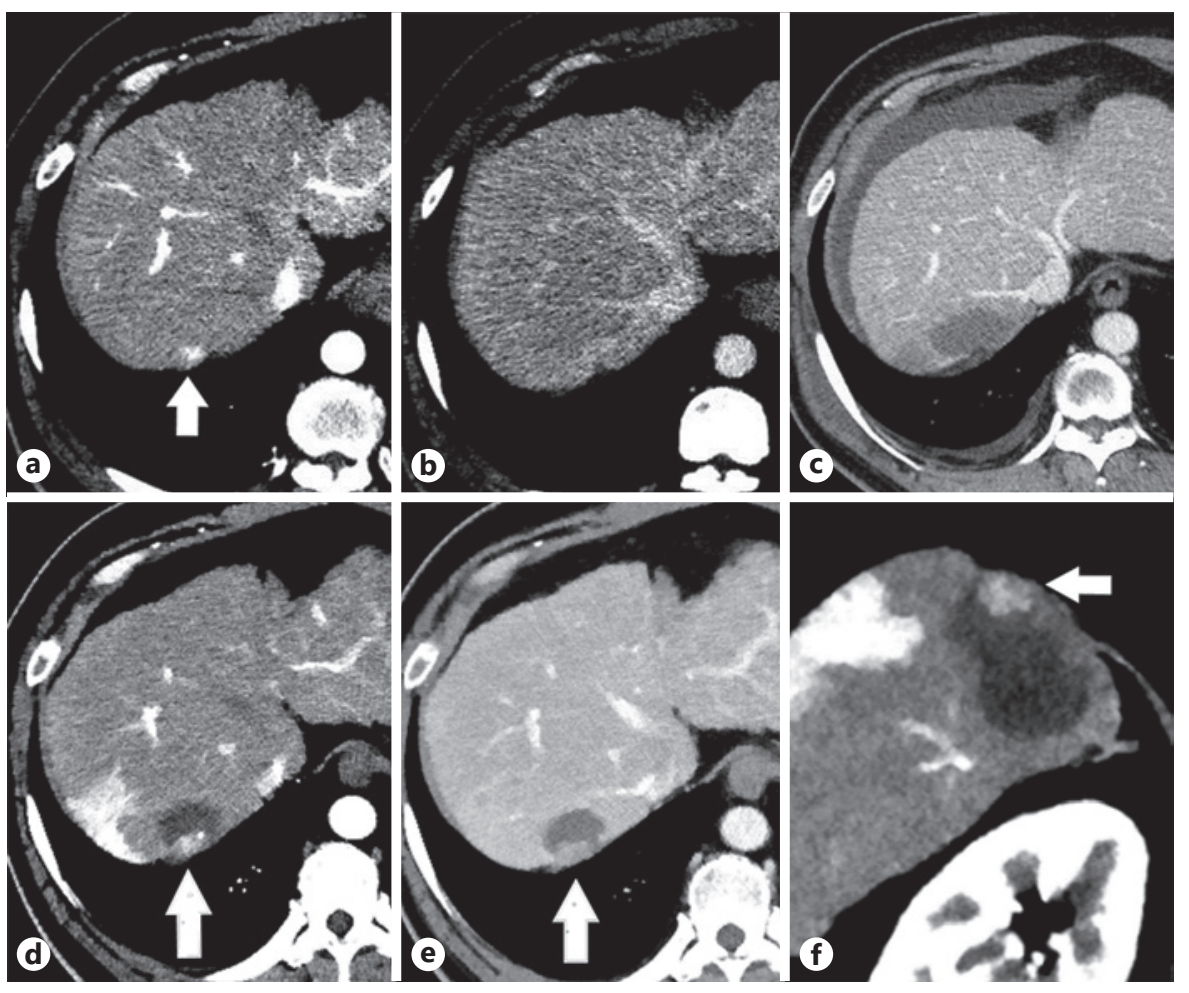

A sufficient ablative margin was achieved in 57 cases out of $60(95 \%)$ in the Nt-RFA group and in 50 cases out of $64(78.1 \%)$ in the conventional RFA group, indicating a statistically significant difference ( $p=0.01$ ).

Both groups did not demonstrate a significant difference in the total procedure time, ablation time, ablation volume, and complications. Thirteen participants demonstrated complications after RFA. In the Nt-RFA group, 7 participants reported complications, the major complication being pseudoaneurysm in 1 case. In conventional RFA, 6 cases were reported, with 1 case being bowel perforation. The intergroup complication rate was not significantly different $(p=0.77)$.

\section{LTP between Conventional RFA and Nt-RFA}

Two participants $(3.4 \%)$ in the Nt-RFA group who achieved treatment success developed LTP (Fig. 4). In the conventional RFA group, 9 participants (15.5\%) developed LTP. Of the 11 participants with LTP, 2 received RFA again and 9 received TACE.

The 1- and 2-year estimated cumulative incidences of LTP were 3.5 and $3.5 \%$ in the Nt-RFA group, and 8.9 and $13.5 \%$ in the conventional RFA group, respectively. The cumulative incidence of LTP in the Nt-RFA group was significantly lower than that in the conventional RFA group ( $p=0.02$ ) (Fig. 5). Predictive factors for the development of LTP are summarized in Table 3. In the multivariable Cox regression analysis, only Nt-RFA was associated with a lower risk of LTP $(\mathrm{p}=0.04$, hazard ratio $=0.2$, $95 \%$ confidence interval $=0.04-0.94)$.

\section{Discussion}

Our results demonstrated that Nt-RFA is more effective at controlling LTP than conventional RFA (2-year LTP rates: $3.5 \%$ in the Nt-RFA group and $13.5 \%$ in the conventional RFA group). The cumulative incidence of LTP was significantly lower in Nt-RFA, and conventional RFA was evaluated as a risk factor for LTP. Our study results were in good agreement with the results of the previous study by Seror et al. [13], which reported enhanced complete necrosis in Nt-RFA (26 out of 29, 89.6\%) using a multibipolar approach compared with conventional RFA (14 out of $30,46.6 \%)$. Similarly, another study by Kawamura et al. [32] also reported 2.9\% LTP rates of NtRFA in comparison with $17.7 \%$ LTP rates of conventional RFA. The better results of Nt-RFA than conventional RFA in lowering LTP rates could be attributed to several technical advantages of Nt-RFA. First, Nt-RFA can first 
Fig. 5. Comparison of survival estimation and cumulative incidence of local tumor recurrence between the two groups.

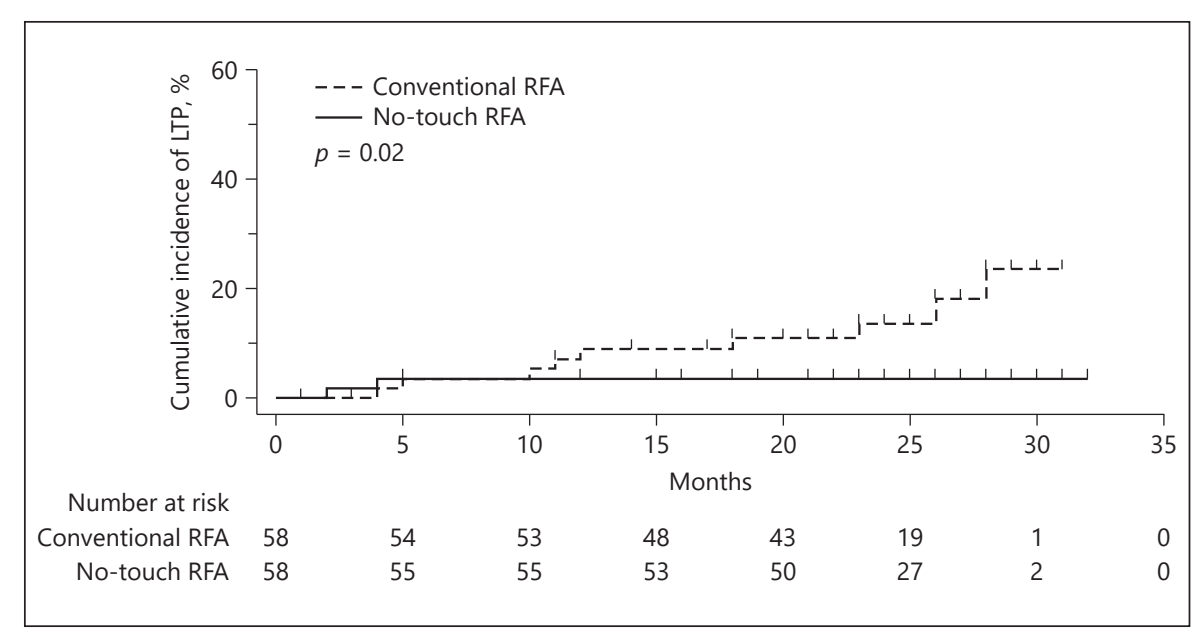

induce vessel coagulation around the tumor, which may possibly result in less intratumoral pressure relative to conventional RFA [14] and lower tumor propagation. Second, Nt-RFA is known to improve the rate of complete necrosis resulting from a larger centripetal ablation volume, inducing sufficient ablative margins. As RFA was not the first-line treatment modality in our study population, we do not make a concrete conclusion whether the development of LTP significantly affected overall patient survival or not. However, according to a recent study by Lee et al. [33] which reported 10-year outcomes of RFA as first-line therapy for single $\mathrm{HCC}<3 \mathrm{~cm}$, LTP was an important prognostic factor for overall survival after percutaneous RFA. So, even if the development of LTP may not significantly affect overall patient survival, we believe that the lower rate of LTP in the Nt-RFA group may contribute to lengthening the recurrence-free survival and lowering the need for additional interventional procedures to obtain a similar overall survival outcome [6].

There have been several studies comparing Nt-RFA and conventional RFA in the treatment of small HCCs showing that Nt-RFA has lower tumor recurrence than conventional RFA, which is consistent with our results [14-16, 34]. Most of these studies were retrospective, with that by Hirooka et al. [16] being a prospective cohort study. In our study, the estimated cumulative incidence of LTP was lower in the Nt-RFA group. LTP typically occurs early and is considered to be related to residual tumor cells in the peritumoral area that have spread microscopically beyond the ablative or resected margin. Therefore, LTP may be more significantly associated with the treatment methodology or result, or the local environment of the tumor such as contact with a vessel. As Nt-
RFA places probes at the surrounding liver parenchyma to the tumor margin without directly puncturing the tumor, there is a reduced risk of direct tumor implantation through the needle or bleeding from the tumor $[11,35$, 36].

In addition, a sufficient ablative margin was more frequently made in Nt-RFA than in conventional RFA: 95\% in the Nt-RFA group and $78.1 \%$ in the conventional RFA group $(p=0.01)$. Interestingly, however, there was no difference in the ablation volume between the two groups, possibly because the needle was moved several times to create a sufficient ablative margin during conventional RFA. Furthermore, with regard to total procedure time and ablation time, there were no significant intergroup differences. The superior results of Nt-RFA appeared to be consistent with the aforementioned principle of NtRFA. Moreover, recent advances in the fusion imaging technique have resulted in improved tumor visibility and technical feasibility when applied to Nt-RFA procedures [22]. This aided with RFA procedure monitoring, making the Nt-RFA technique easier to implement. Although a previous study by Shiina et al. [37] reported that conventional RFA using the multiple overlapping ablation technique with a single electrode provided an excellent LTP rate of $3.2 \%$ at 5- and 10-year follow-ups, these results seemed to be only possible by experienced operators in a high-volume center, and by repeated RFAs until creation of sufficient ablation including tumor and surrounding liver tissue during the hospitalization period. In fact, many other previous studies have reported a $10-30 \%$ recurrence rate after single-session RFA using the multiple overlapping technique [38-41]. The relatively higher recurrence rate of conventional RFA in many previous 
studies could be related to the technical difficulty of precise placement of electrodes in an untreated area of the target tumor (overlapping ablation) in the presence of numerous microbubbles in the treated area following an earlier ablation session on ultrasound guidance [42].

Our study had several limitations. Firstly, our followup duration after RFA (mean 24.1 months) was short. However, since we aimed to evaluate LTP by comparing two techniques of RFA, there was no need for a long-term follow-up. Secondly, the effectiveness of RFA may depend on the operator's experience and skill. RFAs were performed by four radiologists in our study and the results may not be reproducible. This is a general limitation of many clinical studies. Thirdly, we used a switching monopolar mode as the RF energy delivery mode in our study. The previous studies have shown that the multibipolar mode produces centripetal energy, preventing the ablation zone from perfusion-mediated cooling, resulting in more focal heating between the electrodes and being less affected by the heat sink effect [14]. Moreover, according to our previous in vivo experimental study [26], the switching bipolar mode was more advantageous for NtRFA as it could create confluent necrosis more frequently and also had a better safety profile. Indeed, in our study, because the participants had small HCCs with a maximum diameter of $2.5 \mathrm{~cm}$, a switching monopolar mode could be used. Further study is required to determine which RF energy delivery mode is better for improving tumor control rates using a "no-touch" ablation technique. Fourthly, most of the participants had prior HCC treatments. It can interfere with the result such as intrahepatic distant recurrence, recurrence-free survival, or overall survival. So, we focused on LTP to compare two techniques. Despite these limitations, this study is still meaningful given that it is a randomized prospective study.

In conclusion, a switching monopolar Nt-RFA is a favorable treatment modality and provides a lower LTP rate compared with conventional RFA for small HCC. Therefore, it may contribute to lengthening the recurrence-free survival and also decrease the necessity of additional interventional procedures to obtain a similar overall survival outcome compared to conventional RFA.

\section{Statement of Ethics}

This randomized clinical trial was performed from October 2016 to September 2017 in accordance with the ethical guidelines of the 1975 Declaration of Helsinki. Participants have given their written informed consent, and the protocol was approved by the Institutional Review Board of our institution (IRB No. 1604-137758).

\section{Conflict of Interest Statement}

The authors have no conflicts of interest to declare.

\section{Funding Sources}

Financial support was provided by STARmed Corp. (No. 062016-2370).

\section{Author Contributions}

- Sae-Jin Park: data curation, formal analysis, investigation, software, writing original draft.

- Eun Ju Cho, Jeong-Hoon Lee, Su Jong Yu, Yoon Jun Kim, JungHwan Yoon, Hyo-Jin Kang: data curation.

- Jeong Hee Yoon: data curation, writing review and editing.

- Dong Ho Lee: data curation, supervision, writing review and editing.

- Se Hyung Kim, Jae Young Lee: data curation.

- Jeong Min Lee: data curation, supervision, writing review and editing.

\section{References}

1 Fateen W, Ryder SD. Screening for hepatocellular carcinoma: patient selection and perspectives. J Hepatocell Carcinoma. 2017 May; 4:71-9.

2 Feng K, Ma KS. Value of radiofrequency ablation in the treatment of hepatocellular carcinoma. World J Gastroenterol. 2014 May; 20(20):5987-98.

3 Fong ZV, Tanabe KK. The clinical management of hepatocellular carcinoma in the United States, Europe, and Asia: a comprehensive and evidence-based comparison and review. Cancer. 2014 Sep;120(18):2824-38.
4 Ahmed M, Brace CL, Lee FT Jr, Goldberg SN. Principles of and advances in percutaneous ablation. Radiology. 2011 Feb;258(2):351-69.

5 Giorgio A, Di Sarno A, De Stefano G, Scognamiglio U, Farella N, Mariniello A, et al. Percutaneous radiofrequency ablation of hepatocellular carcinoma compared to percutaneous ethanol injection in treatment of cirrhotic patients: an Italian randomized controlled trial. Anticancer Res. 2011 Jun;31(6):2291-5.

6 Lee DH, Lee JM, Lee JY, Kim SH, Yoon JH, Kim YJ, et al. Radiofrequency ablation of hepatocellular carcinoma as first-line treatment: long-term results and prognostic factors in 162 patients with cirrhosis. Radiology. 2014 Mar;270(3):900-9.

7 Mulier S, Mulier P, Ni Y, Miao Y, Dupas B, Marchal G, et al. Complications of radiofrequency coagulation of liver tumours. $\mathrm{Br} \mathrm{J}$ Surg. 2002 Oct;89(10):1206-22.

8 Livraghi T, Solbiati L, Meloni MF, Gazelle GS, Halpern EF, Goldberg SN. Treatment of focal liver tumors with percutaneous radio-frequency ablation: complications encountered in a multicenter study. Radiology. $2003 \mathrm{Feb}$; 226(2):441-51. 
9 Ruzzenente A, Manzoni GD, Molfetta M, Pachera S, Genco B, Donataccio M, et al. Rapid progression of hepatocellular carcinoma after Radiofrequency Ablation. World J Gastroenterol. 2004 Apr;10(8):1137-40.

10 Kotoh K, Morizono S, Kohjima M, Enjoji M, Sakai H, Nakamuta M. Evaluation of liver parenchymal pressure and portal endothelium damage during radio frequency ablation in an in vivo porcine model. Liver Int. 2005 Dec; 25(6): 1217-23.

11 Kotoh K, Nakamuta M, Morizono S, Kohjima M, Arimura E, Fukushima M, et al. A multistep, incremental expansion method for radio frequency ablation: optimization of the procedure to prevent increases in intra-tumor pressure and to reduce the ablation time. Liver Int. 2005 Jun;25(3):542-7.

12 Angonese C, Baldan A, Cillo U, D'Alessandro A, De Antoni M, De Giorgio M, et al. Complications of radiofrequency thermal ablation in hepatocellular carcinoma: what about "explosive” spread? Gut. 2006 Mar;55(3):435-6.

13 Seror O, N'Kontchou G, Van Nhieu JT, Rabahi Y, Nahon P, Laurent A, et al. Histopathologic comparison of monopolar versus notouch multipolar radiofrequency ablation to treat hepatocellular carcinoma within Milan criteria. J Vasc Interv Radiol. 2014 Apr;25(4): 599-607.

14 Hocquelet A, Aubé C, Rode A, Cartier V, Sutter O, Manichon AF, et al. Comparison of notouch multi-bipolar vs. monopolar radiofrequency ablation for small HCC. J Hepatol. 2017 Jan;66(1):67-74.

15 Kawamura Y, Ikeda K, Fujiyama S, Hosaka T, Kobayashi M, Saitoh S, et al. Potential of a notouch pincer ablation procedure that uses a multipolar radiofrequency ablation system to prevent intrasubsegmental recurrence of small and single hepatocellular carcinomas. Hepatol Res. 2017 Sep;47(10):1008-20.

16 Hirooka M, Hiraoka A, Ochi H, Koizumi Y, Michitaka K, Joko K, et al. Prospective cohort trial to confirm the efficacy of no-touch radio frequency ablation. J Gastroenterol Hepatol. 2019 Mar;34(3):567-74.

17 Choi TW, Lee JM, Lee DH, Lee JH, Yu SJ, Kim YJ, et al. Percutaneous Dual-Switching Monopolar Radiofrequency Ablation Using a Separable Clustered Electrode: A Preliminary Study. Korean J Radiol. 2017 Sep-Oct;18(5): 799-808.

18 Mitchell DG, Bruix J, Sherman M, Sirlin CB. LI-RADS (Liver Imaging Reporting and Data System): summary, discussion, and consensus of the LI-RADS Management Working Group and future directions. Hepatology. 2015 Mar;61(3):1056-65.

19 Fasel JH, Schenk A. Concepts for liver segment classification: neither old ones nor new ones, but a comprehensive one. J Clin Imaging Sci. 2013 Oct;3:48.

20 Kang TW, Lim HK, Lee MW, Kim YS, Rhim $\mathrm{H}$, Lee WJ, et al. Long-term Therapeutic Outcomes of Radiofrequency Ablation for Subcapsular versus Nonsubcapsular Hepatocel- lular Carcinoma: A Propensity Score Matched Study. Radiology. 2016 Jul;280(1):300-12.

21 Chang W, Lee JM, Lee SM, Han JK. No-Touch Radiofrequency Ablation: A Comparison of Switching Bipolar and Switching Monopolar Ablation in Ex Vivo Bovine Liver. Korean J Radiol. 2017 Mar-Apr;18(2):279-88.

22 Ahn SJ, Lee JM, Lee DH, Lee SM, Yoon JH, Kim YJ, et al. Real-time US-CT/MR fusion imaging for percutaneous radiofrequency ablation of hepatocellular carcinoma. J Hepatol. 2017 Feb;66(2):347-54.

23 Choi JW, Lee JM, Lee DH, Yoon JH, Suh KS, Yoon JH, et al. Switching Monopolar Radiofrequency Ablation Using a Separable Cluster Electrode in Patients with Hepatocellular Carcinoma: A Prospective Study. PLoS One. 2016 Aug;11(8):e0161980.

24 Chang W, Lee JM, Lee DH, Yoon JH, Kim YJ, Yoon JH, et al. Comparison of switching bipolar ablation with multiple cooled wet electrodes and switching monopolar ablation with separable clustered electrode in treatment of small hepatocellular carcinoma: A randomized controlled trial. PLoS One. 2018 Feb;13(2):e0192173.

25 Patidar Y, Singhal P, Gupta S, Mukund A, Sarin SK. Radiofrequency ablation of surface $\mathrm{v} / \mathrm{s}$ intraparenchymal hepatocellular carcinoma in cirrhotic patients. Indian J Radiol Imaging. 2017 Oct-Dec;27(4):496-502.

26 Chang W, Lee JM, Yoon JH, Lee DH, Lee SM, Lee KB, et al. No-touch radiofrequency ablation using multiple electrodes: an in vivo comparison study of switching monopolar versus switching bipolar modes in porcine livers. PLoS One. 2017 Apr;12(4):e0176350.

27 Lee JM, Han JK, Kim HC, Kim SH, Kim KW, Joo SM, et al. Multiple-electrode radiofrequency ablation of in vivo porcine liver: comparative studies of consecutive monopolar, switching monopolar versus multipolar modes. Invest Radiol. 2007 Oct;42(10):676-83.

28 Lee DH, Lee JM, Lee JY, Kim SH, Kim JH, Yoon $\mathrm{JH}$, et al. Non-hypervascular hepatobiliary phase hypointense nodules on gadoxetic acid-enhanced MRI: risk of HCC recurrence after radiofrequency ablation. J Hepatol. 2015 May;62(5):1122-30.

29 Rathke H, Hamm B, Güttler F, Rathke J, Rump J, Teichgräber U, et al. Comparison of four radiofrequency ablation systems at two target volumes in an ex vivo bovine liver model. Diagn Interv Radiol. 2014 May-Jun;20(3): 251-8.

30 Wang X, Sofocleous CT, Erinjeri JP, Petre EN, Gonen M, Do KG, et al. Margin size is an independent predictor of local tumor progression after ablation of colon cancer liver metastases. Cardiovasc Intervent Radiol. 2013 Feb; 36(1):166-75

31 Seror O, N'Kontchou G, Nault JC, Rabahi Y, Nahon P, Ganne-Carrié N, et al. Hepatocellular Carcinoma within Milan Criteria: NoTouch Multibipolar Radiofrequency Ablation for Treatment-Long-term Results. Radiology. 2016 Aug;280(2):611-21.
32 Kawamura Y, Ikeda K, Shindoh J, Kobayashi Y, Kasuya K, Fujiyama S, et al. No-touch ablation in hepatocellular carcinoma has the potential to prevent intrasubsegmental recurrence to the same degree as surgical resection. Hepatol Res. 2019 Feb;49(2):164-76.

33 Lee MW, Kang D, Lim HK, Cho J, Sinn DH, Kang TW, et al. Updated 10-year outcomes of percutaneous radiofrequency ablation as first-line therapy for single hepatocellular carcinoma $[\{\mathrm{LT}\}] 3 \mathrm{~cm}$ : emphasis on association of local tumor progression and overall survival. Eur Radiol. 2020 Apr;30(4):2391400.

34 Chai Y, Li K, Zhang C, Chen S, Ma K. The short-term efficacy of no-touch radiofrequency ablation in treating cirrhosis-based small hepatocellular carcinoma. BMC Cancer. 2019 May; 19(1):497.

35 Mori Y, Tamai H, Shingaki N, Moribata K, Shiraki T, Deguchi H, et al. Diffuse intrahepatic recurrence after percutaneous radiofrequency ablation for solitary and small hepatocellular carcinoma. Hepatol Int. 2009 Sep 3(3):509-15.

36 Otsuka Y, Kaneko H. Laparoscopic liver resection in the treatment of HCC with liver cirrhosis: would it provide superiority to conventional open hepatectomy? Hepatobiliary Surg Nutr. 2017 Oct;6(5):356-8.

37 Shiina S, Tateishi R, Arano T, Uchino K, Enooku K, Nakagawa H, et al. Radiofrequency ablation for hepatocellular carcinoma: 10 year outcome and prognostic factors. Am J Gastroenterol. 2012 Apr;107(4):569-77.

38 Kobayashi M, Ikeda K, Kawamura Y, Hosaka T, Sezaki H, Yatsuji H, et al. Randomized controlled trial for the efficacy of hepatic arterial occlusion during radiofrequency ablation for small hepatocellular carcinoma-direct ablative effects and a long-term outcome. Liver Int. 2007 Apr;27(3):353-9.

39 Livraghi T, Meloni F, Di Stasi M, Rolle E, Solbiati L, Tinelli C, et al. Sustained complete response and complications rates after radiofrequency ablation of very early hepatocellular carcinoma in cirrhosis: is resection still the treatment of choice? Hepatology. 2008 Jan; $47(1): 82-9$.

40 Gao J, Wang SH, Ding XM, Sun WB, Li XL, $\mathrm{Xin} \mathrm{ZH}$, et al. Radiofrequency ablation for single hepatocellular carcinoma $3 \mathrm{~cm}$ or less as first-line treatment. World J Gastroenterol. 2015 May;21(17):5287-94.

41 Vitali GC, Laurent A, Terraz S, Majno P, Buchs NC, Rubbia-Brandt L, et al. Minimally invasive surgery versus percutaneous radio frequency ablation for the treatment of single small $(\leq 3 \mathrm{~cm})$ hepatocellular carcinoma: a case-control study. Surg Endosc. 2016 Jun; 30(6):2301-7.

42 Dodd GD 3rd, Frank MS, Aribandi M, Chopra S, Chintapalli KN. Radiofrequency thermal ablation: computer analysis of the size of the thermal injury created by overlapping ablations. AJR Am J Roentgenol. 2001 Oct; 177(4):777-82. 